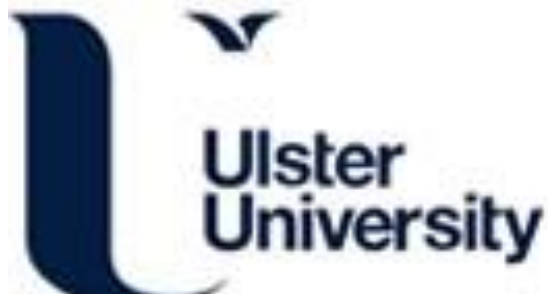

\section{Heartrate Variability Comparison Between Electrocardiogram, Photoplethysmogram and Ballistic Pulse Waveforms at Fiducial Points}

Janjua, G., Hadia, R., Guldenring, D., Finlay, D., \& McLaughlin, J. (2017). Heartrate Variability Comparison Between Electrocardiogram, Photoplethysmogram and Ballistic Pulse Waveforms at Fiducial Points. IFMBE Proceedings, 66, 171-177. https://doi.org/10.1007/978-981-10-7419-6_29

Link to publication record in Ulster University Research Portal

\author{
Published in: \\ IFMBE Proceedings
}

Publication Status:

Published (in print/issue): 17/11/2017

DOI:

10.1007/978-981-10-7419-6_29

\section{Document Version}

Author Accepted version

\section{General rights}

Copyright for the publications made accessible via Ulster University's Research Portal is retained by the author(s) and / or other copyright owners and it is a condition of accessing these publications that users recognise and abide by the legal requirements associated with these rights.

\section{Take down policy}

The Research Portal is Ulster University's institutional repository that provides access to Ulster's research outputs. Every effort has been made to ensure that content in the Research Portal does not infringe any person's rights, or applicable UK laws. If you discover content in the Research Portal that you believe breaches copyright or violates any law, please contact pure-support@ulster.ac.uk. 


\title{
Heartrate variability comparison between Electrocardiogram, Photoplethysmogram and Ballistic pulse waveforms at fiducial points
}

\author{
G. M.W. Janjua, R. Hadia, D. Guldenring, D.D. Finlay, J. A. D. McLaughlin \\ Ulster University, Belfast, United Kingdom
}

\begin{abstract}
Heart rate variability analysis (HRVA) gives valuable insight to the cardiovascular system. Electrocardiogram (ECG) based HRVA has been assessment gold standard but eavesdropping of wearable technology requires the comparison of its surrogacy to an accepted standard. In this study, optical and mechanical measures at distal artery waveform are compared to the electrical signal of the heart. The sensor data of the six healthy volunteers are collated and compared at fiducial points in various time, frequency and non-linear domains for HRVA. We have found that during early systole fiducial location on waveforms can be surrogate to ECG standard and mechanical sensor $2^{\text {nd }}$ derivative proved to be the best among them. Also, the comparative technology shows enormous potential for cardiovascular diagnostic.
\end{abstract}

Keywords - Heartrate variability, Electrocardiogram, Photoplethysmogram, Ballistic pulse pressure, fiducial point.

\section{INTRODUCTION}

Cardiovascular diseases caused by a change in heart rhythm are the leading cause of morbidity and mortality in this modern era, globally. It accounts for approximately $31 \%$ of deaths worldwide [1]. Non-invasive physiological signs, like blood pressure (BP) variation, HRV and arterial stiffness are few of the widely used, as simplest to acquire vital physiological signs in accessing the cardiovascular health [2]. $\mathrm{HRV}$, the change in the time interval between adjacent heartbeats, is one of the most prominent noninvasive markers to establish the meaningful relationship between the autonomic nervous system and cardiovascular mortality, including sudden cardiac deaths [3]. Most commonly used techniques for HRVA is based on RR interval variability (RRIV) on the ECG waveform and by the advent of wearable technology peripheral photoplethysmograph (PPG) pulse rate variability (PRV) analysis is also becoming famous. The most commonly used and 'Gold standard' HRVA measurement method is the RR interval variability measurement from ECG [4]. The rapid increase in aging population and the increase in demand for home healthcare monitoring have increased research interest in wearable healthcare technologies. Within the last decade, many studies have been carried out to realize the real-time acquisition of different physiological parameters using wearable sensors [5]. Also, in recent times, a lot of work has been done in the direction to implement easy to use, noninvasive single acquisition location wearable technologies aimed at assessing and improving cardiovascular health. These works include the development of novel wearable devices to continuously monitor BP variations, HRVA and sleep apnea. In the market currently, many wearable devices are available to monitor HRV based on PRV and RRIV. In this research, we are presenting a comparative analysis of different waveform based HRVA measurement techniques against the RRIV based HRVA measurement. The signals used are, PPG, which works on the optical absorption phenomenon and a poor man tonometry, which measures the mechanical pressure variation of blood in artery, a distension waveform. The pulse wave rate variability based HRVA measurement techniques studied during this research are PPG \& ballistic pulse pressure (BPP) measurement, a poor man tonometer device. The techniques mentioned here used various fiducial points measured on waveform which are the peak, $1^{\text {st }}$ and $2^{\text {nd }}$ derivative. In this experiment, we have used single measurement methods on peripheral pulse which detects the flow pulsation from the blood volume change in the peripheral arteries optically and mechanically, PPG and BPP respectively.

\section{MATERIAL AND METHOD}

For this study, six healthy male volunteers were recruited by age group $34 \pm 9$ years. The subjects were asked to relax for five minutes on an armchair before vital sign data acquisition and informed consent was taken before the study. The Biopac MP36R system was used for simultaneous data acquisition of all signals and subjects were asked to stay calm during the data acquisition to reduce the noise and motion artifacts. The data was sampled at the $2-\mathrm{kHz}$ frequency with a resolution of 24-bit from acquisition unit for data analysis. Three sensors and their placements are: 1) The ECG signal is a gold standard for HRVA and reference for another signal for this comparative study, so to achieve noise-free signals chest position is selected as it provides the most stable location for cardiac activity monitoring. 2) One of the top trends in the medical industry is wristwatch based cardiac activity monitor- due to easy of their used and internet connectivity. 
To mimic this scenario, we have placed a PPG sensor of $960 \mathrm{~nm}$ wavelength on the sensor on subject's wrist position and subjects were asked to refrain from movement to capture noise and motion artifact-free signals. 3) The use of tonometer in the measurement of vascular hemodynamics is very popular in biomedical and clinical research. A poor man tonometer, custom designed device, BPP sensor [10] is placed on a subject palmar artery in index finger to acquire subject's artery distension waveform. The collated dataset was exported in text file format for analysis in MATLAB environment, on a Windows PC.

Table 1 HRVA dataset during resting heart condition

\begin{tabular}{ccc}
\hline BP Start=>Stop & Age & $\mathrm{H}(\mathrm{cm}) / \mathrm{W}(\mathrm{Kg})=>$ BMI \\
\hline $139 / 98=>137 / 96$ & 45 & $175 / 85=>27.76$ \\
$118 / 77=>125 / 77$ & 29 & $177 / 80=>25.54$ \\
$116 / 71=>116 / 73$ & 32 & $174 / 78=>25.76$ \\
$121 / 71=>127 / 72$ & 30 & $180 / 83=>25.62$ \\
$119 / 66=>115 / 73$ & 27 & $188 / 73=>20.65$ \\
$117 / 67=>122 / 68$ & 29 & $179 / 84=>26.22$ \\
\hline
\end{tabular}

\section{FidUCIAL POINTS SELECTION}

We have selected three key locations for our analysis and they are compared against the RR HRVA of ECG and these fiducial points are described:

1. The peaks of waveform

2. The peaks of $1^{\text {st }}$ derivative of waveform

3. The Peak of $2^{\text {nd }}$ derivative of waveform

The significance of these markers has been reported in the literature [10]. During the blood flow in the arterial tree, some of these points are greatly affected by wave reflection due to bifurcation and peripheral resistance. One of the purposes of this study assesses the effect of wave reflection and to highlight the importance of wave reflection; for example, the peak of the waveform is greatly affected by a reflected wave in hypertensive subjects [10]. Whereas, waveforms are reflection free in early systole and $1^{\text {st }}$ and $2^{\text {nd }}$ derivatives are the critical points in these aspects. The foot of the waveform is also least affected by wave reflection but it can be impaired by technological limitation like sink color, hairs, and contact to the body in wearable sensors. The intersecting tangent has proved to be a crucial point due to reproducibility and resilience to artifacts but doesn't have physiological interpretation. The selected location of fiducial points is shown along with waveforms in figure 1.
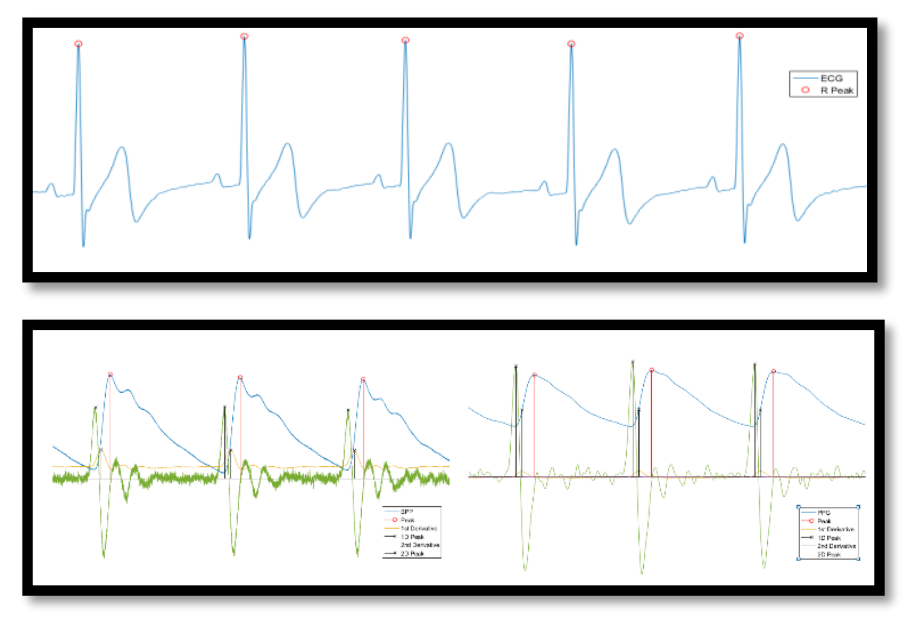

Figure 1: Waveforms and fiducial points

\section{HRV ALGORITHM}

The HRV is compared across thefollowing aspect:

1. Time domain

a. Standard deviation of the normal-to-normal $(\mathrm{NN})$ interval series (SDNN)

b. Root mean square of successive differences (RMSSD)

c. Triangular interpolation of the $\mathrm{NN}$ interval (TINN)

\section{Frequency domain}

a. Power spectrum density (PSD)

i. VLF, LF, and HF frequency 0.003$0.04 \mathrm{~Hz}, \quad 0.04-0.15 \mathrm{~Hz}$, and $0.15-0.4 \mathrm{~Hz}$ respectively

3. Time-frequency domain

a. Windowed Periodogram

b. Continuous Wavelet Transform

c. Discrete Wavelet Transform

4. Nonlinear domain
a. Poincare plot analysis
b. Sample Entropy
c. Detrended Fluctuation Analysis

The open source code [6] has been used due to ease of customization and to test calculate HRVA on the above-mentioned domain. The data is preprocessed by fourth order butter worth filter to remove noise from the signals and fiducial points were taken by taking the peaks of waveform and peaks of $1^{\text {st }}$ and $2^{\text {nd }}$ derivative waveforms. The R-peak on ECG signal is detected using Pans-Tompkins algorithm and data was visually inspected for cardiac arrhythmia. All fiducial points were detected and beat to beat interval is calculated for HRVA. 


\section{RESUTLS AND DISCUSSION}

The cardiac chronotropic modulation by autonomic nerves system facilitates end-organ perfusion in coordination to changing blood hemodynamics in arteries, to accommodate the sudden changes in the human circulation system. HRVA is used by biomedical researchers and clinicians to monitor cardiac disease like heart failure, respiratory sinus arrhythmia (RSA), hyperaldosteronism etc. HRVA publicly available resources are Kubios [6], ECGLab [7], KARDIA [8], and PhysioToolkit [9] from which we have selected the HRVAS [10] due to its easy customization. In this study, sensors are compared in the context of e-Health applications as an alternative to chest based ECG systems. The HRVA is conducted on 17 PC in MATLAB environment. The ECG, PPG and BPP waveforms and their fiducial locations based beat to beat interval are shown as below: For agreement analysis, Bland Altman (BA) plot, and regression fit (R-fit) plotted is on following key parameters in respective domains, shown in table 2 . $(\mathrm{pNNx}=$ percentage of successive normal cardiac inter beat intervals, $\mathrm{sdHR}=$ standard deviation Heart rate, $\mathrm{HRVTi}=$ HRV time index, p=peak, VL=very low, L=low, H=high, $\mathrm{F}=$ frequency, SampEn=Sample Entropy, alpha, alpha1 \& alpha2=Short term scaling exponent, SD1= dispersion standard deviation perpendicular to the axis, $\mathrm{SD} 2=$ dispersion standard deviation along the axis)

Table 2 Parameter used for analysis

\begin{tabular}{|c|c|c|c|c|c|}
\hline \multicolumn{6}{|c|}{ Time Domain } \\
\hline SDNN & $\mathrm{pNNx}$ & RMSSD & sdHR & HRVTi & TINN \\
\hline$(\mathrm{ms})$ & $(\%)$ & $(\mathrm{ms})$ & (bpm) & $(\mathrm{ms})$ & $(\mathrm{ms})$ \\
\hline \multicolumn{6}{|c|}{ Freq Domain: Welch periodogram } \\
\hline \multicolumn{6}{|c|}{ Freq Domain: Auto-Regressive periodogram } \\
\hline \multicolumn{6}{|c|}{ Freq Domain: Lomb-Scargle Periodogram } \\
\hline pVLF & $\mathrm{pLF}$ & $\mathrm{pHF}$ & VLF & LF & $\mathrm{HF}$ \\
\hline & $(\%)$ & & & $\operatorname{Peak}(\mathrm{Hz})$ & \\
\hline \multicolumn{4}{|c|}{ Nonlinear } & \multicolumn{2}{|c|}{ Poincare } \\
\hline SampEn & alpha & alpha1 & alpha2 & SD1 & SD2 \\
\hline & & & & & \\
\hline \multicolumn{6}{|c|}{ Time-Freq: Auto-Regressive } \\
\hline \multicolumn{6}{|c|}{ Time-Freq: Lomb Periodogram } \\
\hline \multicolumn{6}{|c|}{ Time-Freq: Wavelet Transform } \\
\hline pVLF & $\mathrm{pLF}$ & $\mathrm{pHF}$ & VLF & LF & $\mathrm{HF}$ \\
\hline & $(\%)$ & & & Peak $(\mathrm{Hz})$ & \\
\hline
\end{tabular}

The RR interval based HRVA of all subjects is tested for an agreement for HRVA for all fiducial points. Plots of Bland Altman analysis are shown below:

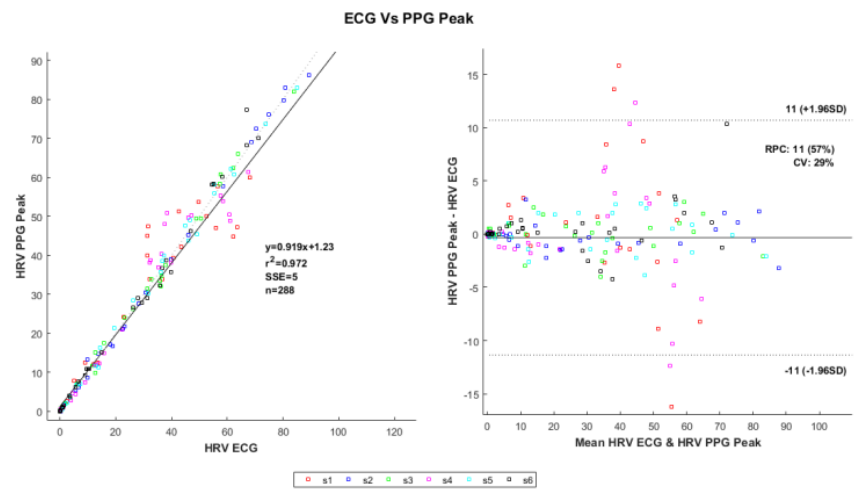

Figure 2: R-fit(left) and BA(right) for ECG RR vs PPG Peak for HRVA

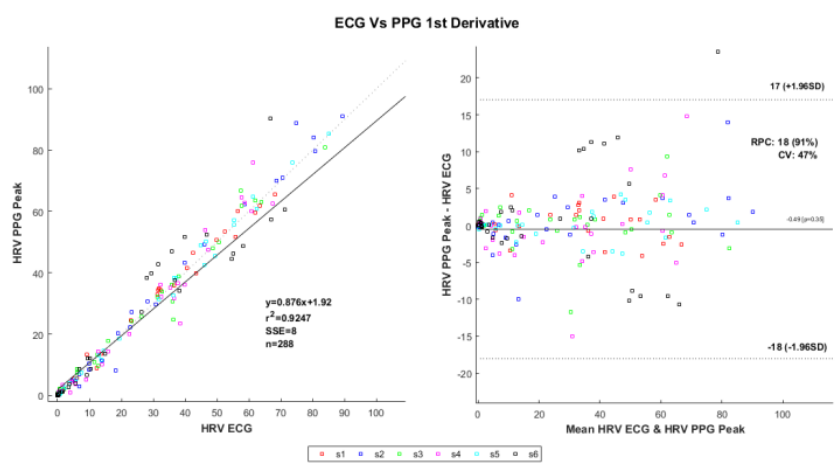

Figure 3: R-fit(left) and BA(right) for ECG RR vs PPG $1^{\text {st }}$ Derivative for HRVA

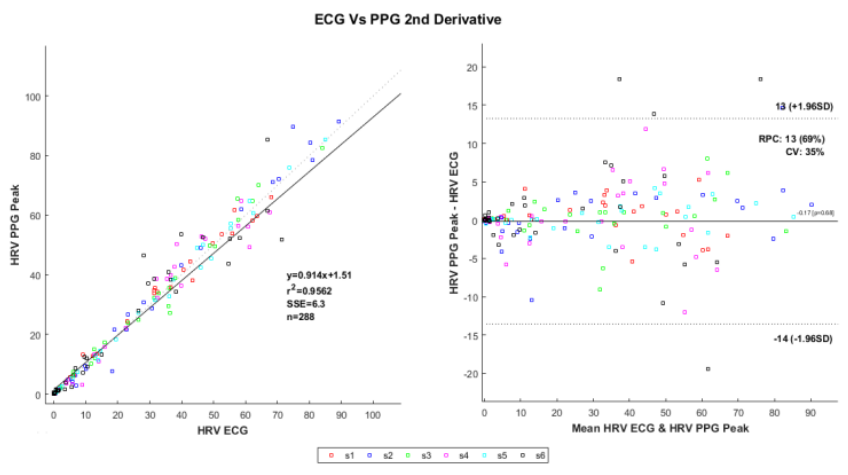

Figure 4: R-fit(left) and BA(right) for ECG RR vs PPG $2^{\text {nd }}$ Derivative for HRVA 


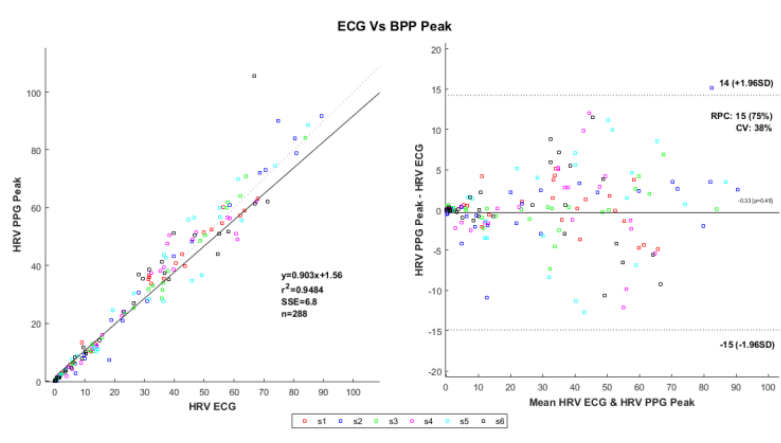

Figure 5: R-fit(left) and BA(right) for ECG RR vs BPP Peak for HRVA

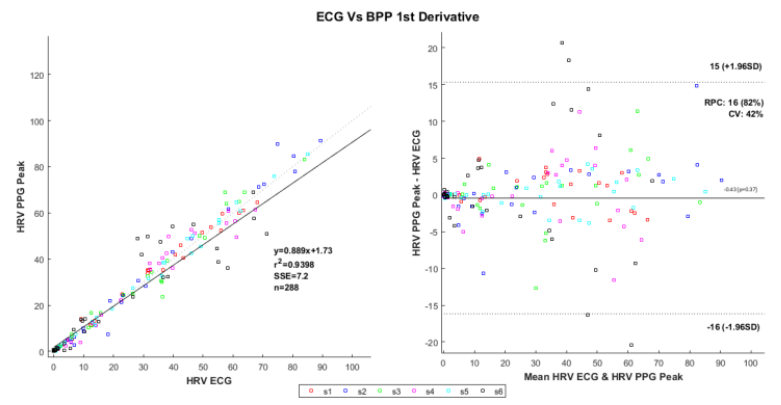

Figure 6: Bland Altman for ECG RR Vs BPP $1^{\text {st }}$ Derivative for HRVA

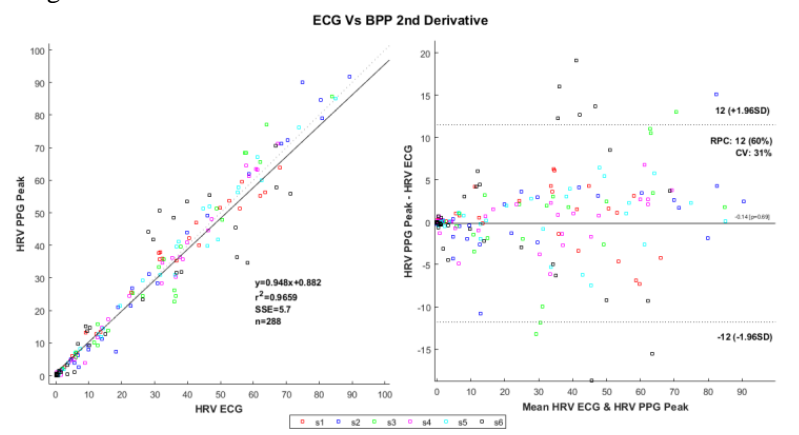

Figure 7: R-fit(left) and BA(right) for ECG RR vs BPP $2^{\text {nd }}$ Derivative for HRVA

We have found correlation information which shows that the overall highest R-square parametric fit is found in PPG peak and BPP $2^{\text {nd }}$ Derivative compared to ECG RR HRVA and lowest found in PPG $1^{\text {st }}$ derivative vice versa for the sum of square error. Also, we have found, BA information, that reproducibility and variation coefficient (RPC \& CV) is lowest in PPG Peak and BPP $2^{\text {nd }}$ Derivative, results shown in table 3 as:

Table 3 R-square fit and BA results

\begin{tabular}{ccccccc}
\hline Marker & PPG_P & PPG_1D & PPG_2D & BPP_P & BPP_1D & BPP_2D \\
\hline $\mathrm{r}^{2}$ & 0.972 & 0.925 & 0.956 & 0.948 & 0.939 & 0.966 \\
RPC/CV & $11 / 29$ & $18 / 47$ & $13 / 35$ & $15 / 38$ & $16 / 42$ & $12 / 31$ \\
\hline
\end{tabular}

\section{CONCLUSIONS}

In this comparative study, we have found that BPP $2^{\text {nd }}$ derivative and PPG peak can be used as a surrogate for ECG RR HRVA. Also, BPP, novel sensor, showed competitive effectiveness to the optical sensor for HRVA.

\section{ACKNOWLEDGMENT}

This project has received funding from the European Union's Horizon 2020 research and innovation program under the Marie Sklodowska-Curie grant agreement No. 676201.

\section{CONFLict OF INTEREST}

The authors declare that they have no conflict of interest.

\section{REFERENCES}

1. Task Force of the European Society of Cardiology the North American Society of Pacing Electrophysiology, "Heart Rate Variability", Circulation, vol. 93, no. 5, p. 1043 LP-1065, Mar. 1996.

2. H. V. Huikuri, T. Makikallio, K. E. J. Airaksinen, R. Mitrani, A. Castellanos, and R. J. Myerburg, "Measurement of heart rate variability: A clinical tool or a research toy?", J. Am. Coll. Cardiol., vol. 34, no. 7, pp. 1878-1883, 1999.

3. L. Politano, A. Palladino, G. Nigro, M. Scutifero, and V. Cozza, Acta Myol., vol. 27, no. 3, pp. 114-122, Dec. 2008.

4. V. Jeyhani, S. Mahdiani, M. Peltokangas, and A. Vehkaoja, "Comparison of HRV parameters derived from photo plethysmography and electrocardiography signals", Proc. Annu. Int. Conf. IEEE Eng. Med. Biol. Soc. EMBS, vol. 2015-November, pp. 5952-5955, 2015.

5. B. D. Nenova, I. T. Iliev, "Non-Invasive Methods of Peripheral Pulse Detection: Advantages and Disadvantages", Annual journal of electronics, pp. 57-60, 2009.

6. J. L. A. de Carvalho, et al., "Development of a Matlab software for analysis of heart rate variability," in Signal Processing, $6^{\text {th }}$ International Conference on, 2002, pp. 1488-1491 vol.2.

7. P. Perakakis, et al., "KARDIA: A Matlab software for the analysis of cardiac interbeat intervals", Comput Methods Programs Biomed, vol. 98, pp. 83-89, 2010.

8. A. L. Goldberger, et al., "PhysioBank, PhysioToolkit, and Physio Net: Components of a New Research Resource for Complex Physiologic Signals," Circ., vol. 101, pp. 215-220, Jun 2000.

9. J. T. Ramshur "Design, evaluation, and application of heart rate variability analysis software “, July 2010.

10. G. Janjua, J. Mclaughlin, D. Finlay, and D. Guldenring, "Wireless Chest Wearable Vital Sign Monitoring Platform", 39th Annual International Conference of the IEEE, EMBC 2017.

Author: Ghalib Muhammad Waqas Janjua

Institute: Ulster University

Street: Shore

City: Belfast

Country: United Kingdom

Email: g.janjua@ulster.ac.uk 